\title{
IVIG Treatment and Prognosis in Guillain-Barré Syndrome
}

\author{
Pieter A. van Doorn • Krista Kuitwaard • \\ Christa Walgaard • Rinske van Koningsveld • \\ Liselotte Ruts $\cdot$ Bart C. Jacobs
}

Published online: 16 April 2010

(C) The Author(s) 2010. This article is published with open access at Springerlink.com

\begin{abstract}
Introduction Guillain-Barré syndrome (GBS) is an acute, immune-mediated polyneuropathy that often leads to severe weakness. Intravenous immunoglobulin (IVIG) is a proven effective treatment for GBS (class 1 evidence). However, about $25 \%$ of patients need artificial ventilation and $20 \%$ are still unable to walk unaided after 6 months. Important clinical factors associated with poor outcome are age, presence of preceding diarrhea and the severity of disability in the early course of disease. These clinical factors were combined in a clinical prognostic scoring scale, the Erasmus GBS Outcome Scale (EGOS).

Materials and Methods GBS patients being unable to walk unaided are currently treated with a standard single IVIg dose $(0.4 \mathrm{~g} / \mathrm{kg}$ bodyweight for 5 days $)$. A recent retrospective study in 174 GBS patients enrolled in one of our randomized controlled clinical trials showed that patients
\end{abstract}

\section{P. A. van Doorn $(\bowtie)$}

Department of Neurology, Erasmus MC,

University Medical Center Rotterdam,

room BA 450, 's-Gravendijkwal 230,

3015 CJ Rotterdam, The Netherlands

e-mail: p.a.vandoorn@erasmusmc.nl

K. Kuitwaard $\cdot$ C. Walgaard $\cdot$ L. Ruts $\cdot$ B. C. Jacobs

Department of Neurology, Erasmus MC,

University Medical Center Rotterdam,

's-Gravendijkwal 230,

3015 CJ Rotterdam, The Netherlands

R. van Koningsveld

Elkerliek Ziekenhuis,

Helmond, The Netherlands

B. C. Jacobs

Department of Immunology, Erasmus MC, University Medical

Center Rotterdam,

Rotterdam, The Netherlands with a minor increase of serum $\operatorname{IgG}$ level after standard single IVIg dose recovered significantly slower. Additionally, fewer patients reached the ability to walk unaided at six months after correction for the known clinical prognostic factors (multivariate analysis; $P<0.022$ ).

Discussion It is yet unknown why some GBS patients only have a minor increase after standard IVIg treatment. By using the EGOS it is possible to select GBS patients with a poor prognosis. These patients potentially may benefit from a second IVIg dose.

Conclusion A standard dose of IVIG is not sufficiently effective in many GBS patients. Whether these patients might benefit from a second IVIg dose needs further investigation.

Keywords Guillain-Barré syndrome · IVIG · treatment . prognosis

\section{Introduction}

Guillain-Barré syndrome (GBS) is a frequent cause of neuromuscular paralysis occurring at all ages. The incidence of GBS is reported to be $1.2-2.3$ per 100,000 per year $[1,2]$. GBS is a postinfectious disorder. The most frequently identified preceding infection is Campylobacter jejuni. Others are cytomegalovirus, Epstein-Barr virus, Mycoplasma pneumoniae, and Haemophilus influenzae [1, 3]. Many reports have documented the occurrence of GBS shortly after vaccinations, operations, or stressful events, but the causality and pathophysiology are still debated [2].

\section{Main Features of GBS}

The main features of GBS are rapid progressive bilateral and relative symmetrical weakness of the limbs with or without 
Table 1 Diagnosis of GuillainBarré Syndrome

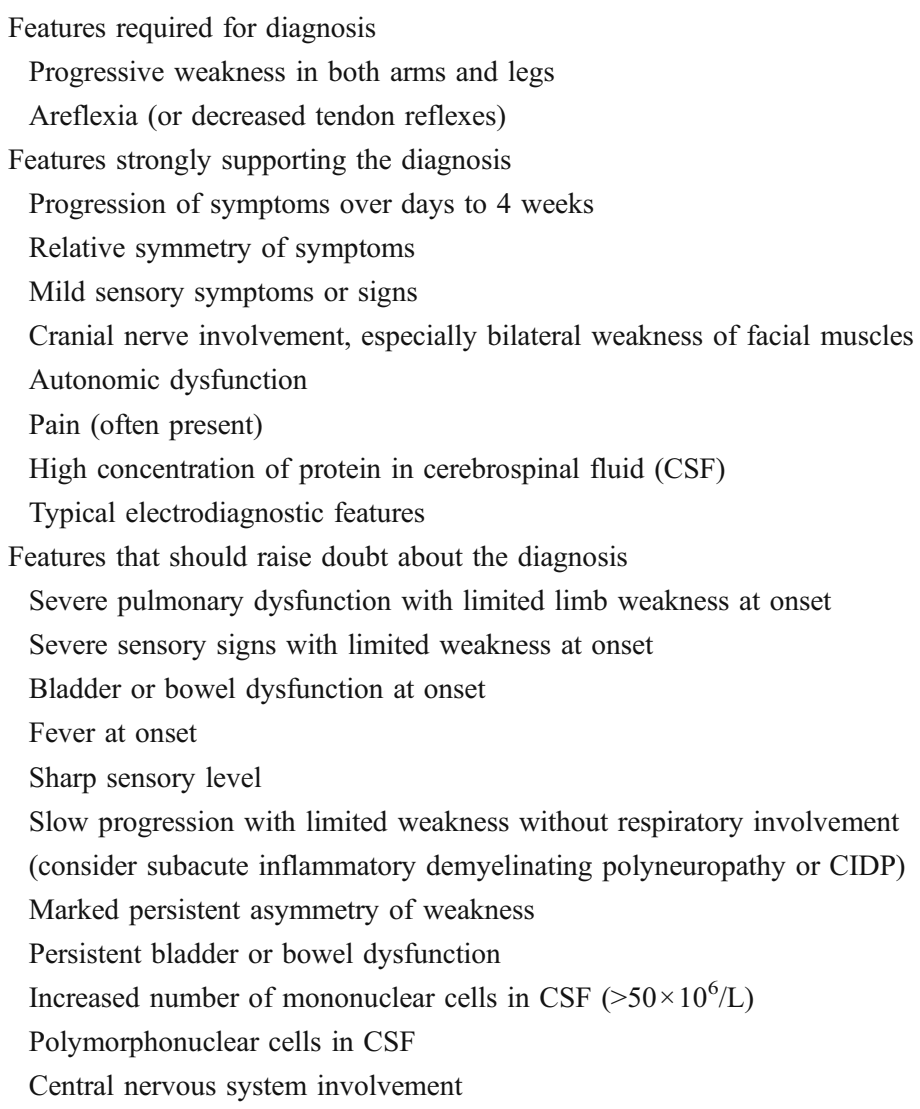

involvement of respiratory or cranial nerve-innervated muscles or sensory disturbances [4]. Patients have decreased or absent tendon reflexes. Cerebrospinal fluid examination typically shows an increased protein level with a normal white cell count. Pain frequently occurs and may cause severe complaints. It often starts before the onset of weakness and therefore can lead to diagnostic difficulties. Diagnostic criteria for GBS are shown in the Table 1. Electromyography (EMG) can be helpful in confirming the diagnosis in clinically difficult cases such as in patients with extreme pain. EMG is especially useful for subclassifying GBS into subgroups such as acute motor axonal neuropathy (AMAN) and acute inflammatory demyelinating polyneuropathy (AIDP).

\section{Clinical Course of GBS}

Rapidly progressive weakness is the core clinical feature of GBS. By definition, maximal weakness is reached within 4 weeks, but most patients reach it within 2 to 3 weeks. Thereafter, patients enter a plateau phase that ranges from days to several weeks or months (Fig. 1). This phase is followed by a usually much slower and variable recovery phase. In Europe, about one-third of GBS patients remain able to walk ("mild patients")
[2]; about $25 \%$ of the GBS patients who are unable to walk ("severe patients") need artificial ventilation. This is predominantly due to weakness of the respiratory muscles. Despite standard treatment with intravenous immunoglobulin (IVIG) or plasma exchange (PE) treatment, about $20 \%$ of severely affected patients remain unable to walk after 6 months. Moreover, many patients remain otherwise disabled or severely fatigued. Even 3 to 6 years after onset, GBS has a great impact on social life and the ability to perform activities of daily life. Therefore, GBS remains a severe disease for which better treatments are required.

\section{Immunobiology}

There is convincing evidence that GBS at least in some patients is caused by an infection-induced aberrant immune response that damages the peripheral nerves. Four key factors were identified that control this process $[1,2,5]$.

1. Anti-ganglioside antibodies

In up to $50 \%$ of patients, serum antibodies to various gangliosides present in human peripheral nerves, including GM1, GD1a, GalNAc-GD1a, and GQ1b, can be demon- 


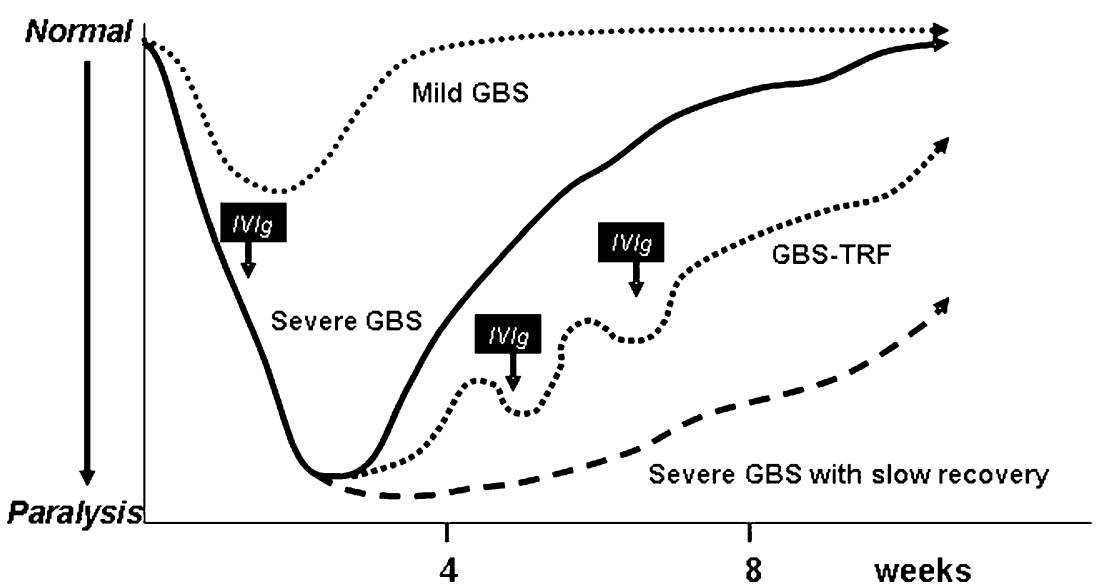

Fig. 1 Variations in course of disease in GBS. Indicated are the courses of disease in mildly and severely affected GBS patients. The effect of IVIG in GBS has only been investigated in randomized controlled trials in patients unable to walk unaided at nadir (severely affected patients) and not in mildly affected patients (able to walk unaided at nadir). Whether mildly affected GBS patients may also benefit from IVIG is yet unknown. GBS patients who initially

strated. Other antibodies may bind to mixtures or complexes of different gangliosides instead of individual ones. Interestingly, most of these antibodies are related to defined clinical subgroups of GBS.

\section{Molecular mimicry and cross-reactivity}

C. jejuni isolates from GBS patients express lipooligosaccharides (LOS) that mimic the carbohydrates of gangliosides. The type of ganglioside mimic in $C$. jejuni seems to determine the specificity of the anti-ganglioside antibodies and the associated variant of GBS. Antibodies in these patients usually are cross-reactive; they recognize LOS as well as gangliosides or ganglioside complexes.

GBS after Campylobacter infection in anti-GM1/GD1a/ GQ1b antibody-related cases is considered to be true example of molecular mimicry-related disease.

\section{Complement activation}

Postmortem studies demonstrated that local complement activation occurs at the side of nerve damage. A mice model for GBS showed that some anti-ganglioside antibodies are toxic for peripheral nerves and can cause blockade of nerve transmission and paralysis of the nerve-muscle preparation. Additionally, there is destruction of the nerve terminal and perisynaptic Schwann cells [6]. Antibodies to GM1 affect the sodium channels at the nodes of Ranvier of rabbit peripheral nerves [7]. All of these effects appear to be dependent on complement activation and formation of the membrane attack complex. The neurotoxic effects of these antibodies can be inhibited by IVIG and the complement inhibitor eculizumab [8]. improve or stabilize after IVIG and subsequently deteriorate again have a "treatment-related fluctuation" (GBS-TRF): a condition that usually responds to an additional IVIG dose. Some GBS patients have a severe course of disease and a slow recovery phase. The prognosis of GBS patients can be determined using the Erasmus GBS Outcome Scale. Whether a second IVIG dose is effective in patients with a poor prognosis is not known yet

\section{Host factors}

Less than 1:1,000 patients with a $C$. jejuni infection will develop GBS. Host factors may influence this susceptibility to develop GBS or the extent of nerve damage and outcome. Single nucleotide polymorphisms (SNPs) showed no consistent association with the susceptibility to develop GBS. Evidence indicates, however, that these SNPs may be important as disease-modifying factors. An association has been demonstrated between disease severity or outcome and SNPs in genes encoding for mannose-binding lectin, Fc $\gamma$ RIII, MMP9, and TNF- $\alpha$ [2]. However, confirmation in more extensive studies is required.

\section{Treatment}

Randomized controlled trials (RCTs) have shown that PE and IVIG are effective in patients with GBS [9, 10]. Most RCTs have used the GBS disability scale to determine the effect of therapy. The GBS disability scale is a 7-point scale, ranging from no symptoms $(F=0)$, the ability to walk $10 \mathrm{~m}$ without assistance $(F=2)$, bedbound $(F=4)$, to death $(F=6)$. The first RCT on the use of IVIG in GBS was published in 1992 and demonstrated that IVIG is as effective as PE [11]. IVIG is applied in a regimen of $2 \mathrm{~g} /$ $\mathrm{kg}$ bodyweight, usually as $\quad 0.4 \mathrm{~g} / \mathrm{kg}$ bodyweight per day for five consecutive days. IVIG has replaced PE as the preferred treatment in many centers, mainly because of its greater convenience and availability [9]. The combination of PE followed by IVIG was not significantly better than 
PE or IVIG alone [12]. It appeared that oral steroids and, surprisingly, intravenous methylprednisolone $500 \mathrm{mg} / \mathrm{day}$ for five consecutive days alone is not beneficial in GBS [13]. The combination of IVIG and intravenous methylprednisolone was not significantly more effective than IVIG alone. Although when a correction was made for known prognostic factors, the combined treatment shows some short-term effect [14]. These trials show that there is an effect of immunotherapy on the course of GBS, but new studies with different regimens or another therapy that is directed toward improving the prognosis and outcome of GBS are needed.

\section{Prognosis of GBS}

The clinical course of GBS in individual patients is highly variable and difficult to predict. Advanced age is generally reported to be a negative prognostic factor. Previous studies indicate that the difference in severity of GBS can be determined in an early phase of the disease [15]. Peroneal nerve conduction block and age above 40 years turned out to be independent predictors of disability at 6 months [16]. We have developed a simple clinical prognostic scoring system that accurately predicts the chance of independent walking after 6 months. The Erasmus GBS Outcome Scale (EGOS) can be easily calculated after the first 2 weeks from disease onset using age, presence of preceding diarrhea, and GBS disability score [17]. Based on the EGOS, the predicted chance to recover to independent walking after 6 months ranges from $1 \%$ to $83 \%$. The accuracy of the scale was confirmed in an independent cohort of GBS patients. The EGOS can be used to inform individual patients about their prognosis, additionally it can be used in new treatment trials that more specifically target GBS patients with poor prognosis. A slightly modified EGOS that can calculate the prognosis only 1 week after hospital admission is currently being constructed.

Standard-Dose IVIG is not Sufficient in some GBS Patients

There is circumstantial evidence that standard IVIG dose ( $2 \mathrm{~g} / \mathrm{kg}$ bodyweight) is not, or not sufficiently, effective in some GBS patients because a proportion of GBS patients continues to deteriorate after a standard course of IVIG [13]. About $8 \%$ to $16 \%$ of GBS patients treated with standard-dose IVIG deteriorate again after initial improvement or stabilization. These patients have a deterioration "treatment-related fluctuation," in which muscle force usually improves again after a repeated dose of IVIG [13]. In addition, the results of a noncontrolled study in a small series of patients suggest that a repeated dose of IVIG may be effective in severe, unresponsive GBS patients [18].
The results of a recent pharmacokinetic study suggest that a minor increase in serum IgG level after standard-dose IVIG is associated with a poor outcome [19].

\section{Pharmacokinetics of IVIG and Outcome}

In a recent study by Kuitwaard et al., serum $\mathrm{IgG}$ levels from 174 GBS patients were determined in samples obtained pretreatment, and at 2 weeks, 4 weeks, 3 months, and 6 months after treatment [19]. The variation in serum IgG levels was most pronounced in the samples at 2 weeks and to a lesser extent at 4 weeks after IVIG treatment. The IgG level at 2 weeks showed a weak correlation with baseline IgG level. To investigate the pharmacokinetics of IVIG, the change in $\operatorname{IgG}$ was calculated by subtracting the baseline level from the level at 2 weeks $(\Delta \mathrm{IgG})$. There was a large variability in $\Delta \mathrm{IgG} 2$ weeks after IVIG treatment. It turned out that patients with a low $\Delta \operatorname{IgG}$ (lower quartiles) had a significantly prolonged time to reach GBS disability score $\leq 2$ (able to walk independently; log-rank $P<0.001$ ). Using multivariate logistic regression, it was shown that baseline IgG levels or IgG levels at 2 weeks were not significantly associated with outcome. However, the increase in $\mathrm{IgG}$ level 2 weeks after standard IVIG $(\Delta \mathrm{IgG})$ was associated with outcome $(P=0.027)$. It appeared that patients with a $\Delta \mathrm{IgG}<3.99 \mathrm{~g} / \mathrm{L}$ were four times less likely (OR 0.25 ) and patients with a $\Delta \mathrm{IgG}$ of 3.99 to $7.30 \mathrm{~g} / \mathrm{L}$ were three times less likely (OR $0.26,9)$ to walk unaided after 6 months than patients with a $\Delta \operatorname{IgG}>10.92 \mathrm{~g} / \mathrm{L}$. This is the first study to show that a high increase in IgG levels after IVIG treatment for GBS is associated with a better prognosis.

\section{Discussion}

IVIG is a proven effective treatment for GBS. Not all patients, however, recover enough after a standard IVIG dose. Several clinical factors are associated with the outcome. Importantly, a low increase of serum IgG levels after a standard IVIG dose appeared to be significantly associated with slower recovery and a worse prognosis. The reason why there is such a large interindividual difference in IgG increase after IVIG treatment is not yet known. The disease severity at the time of IVIG treatment appears to influence the increase in serum IgG level. The lowest increase in $\operatorname{IgG}$ was found in patients with more extensive disability and weakness, as defined by the GBS disability score and the Medical Research Council (MRC) sum score. It is possible that more extensive immune-mediated nerve damage results in an increase in $\operatorname{IgG}$ extravasation into inflamed tissues or a higher catabolic rate. These issues need to be studied further. 
IVIG is an expensive treatment that may induce (generally minor) side effects and is currently not indicated (proven to be effective) in mildly affected GBS patients. A second IVIG dose potentially seems to be indicated in patients with a poor prognosis.

Factors associated with outcome after 6 months are the age of the patient, the presence of preceding diarrhea, and GBS disability score or MRC sum score 1 to 2 weeks after hospital admission; in addition, a factor to be included now seems to be the magnitude in increase of IgG levels 2 weeks after the start of IVIG [17, 19, 20]. Using these clinical factors, it is possible to select a group of GBS patients with a poor prognosis early in the course of the disease. An international trial conducted by the Inflammatory Neuropathy Consortium to study the effect of a second IVIG dose in patients with a poor prognosis based upon the EGOS is expected to start soon.

Conflict of Interest B.C. Jacobs received an honorarium from Baxter for consultancy.

P.A. van Doorn received an unrestricted departmental research grant from Baxter to conduct a randomized controlled trial comparing Gammagard S/D with Kiovig in CIDP, and to conduct an RCT comparing Gammagard S/D with or without methylprednisolone in GBS. He also has received personal and departmental payments for consultancy/RCT board participation from Talecris Biotherapeutics, ZLB Plasma Germany, Baxter, and Octapharma AG.

The other authors have no conflicts of interest to disclose.

Open Access This article is distributed under the terms of the Creative Commons Attribution Noncommercial License which permits any noncommercial use, distribution, and reproduction in any medium, provided the original author(s) and source are credited.

\section{References}

1. Hughes RA, Cornblath DR. Guillain-Barré syndrome. Lancet. 2005;366:1653-66.

2. van Doorn PA, Ruts L, Jacobs BC. Clinical features, pathogenesis, and treatment of Guillain-Barré syndrome. Lancet Neurol. 2008;7:939-50.

3. Jacobs BC, Rothbarth PH, van der Meché FG, Herbrink P, Schmitz PI, de Klerk MA, et al. The spectrum of antecedent infections in GuillainBarré syndrome: a case-control study. Neurology. 1998;51:1110-5.

4. Asbury AK, Cornblath DR. Assessment of current diagnostic criteria for Guillain-Barré syndrome. Ann Neurol. 1990;27:S21-4.

5. Willison HJ. The immunobiology of Guillain-Barré syndromes. J Peripher Nerv Syst. 2005;10:94-112.
6. Halstead SK, O'Hanlon GM, Humphreys PD, Morrison DB, Morgan BP, Todd AJ, et al. Anti-disialoside antibodies kill perisynaptic Schwann cells and damage motor nerve terminals via membrane attack complex in a murine model of neuropathy. Brain. 2004;127:2109-23.

7. Susuki K, Rasband MN, Tohyama K, Koibuchi K, Okamoto S, Funakoshi K, et al. Anti-GM1 antibodies cause complementmediated disruption of sodium channel clusters in peripheral motor nerve fibers. J Neurosci. 2007;27:3956-67.

8. Halstead SK, Zitman FM, Humphreys PD, Greenshields K, Verschuuren JJ, Jacobs BC, et al. Eculizumab prevents antiganglioside antibody-mediated neuropathy in a murine model. Brain. 2008;131:1197-208.

9. Hughes RA, Raphael JC, Swan AV, van Doorn PA. Intravenous immunoglobulin for Guillain-Barré syndrome. Cochrane Database Syst Rev. CD002063, 2006.

10. Raphael JC, Chevret S, Hughes RA, Annane D. Plasma exchange for Guillain-Barré syndrome. Cochrane Database Syst Rev. CD001798, 2002.

11. van der Meché FG, Schmitz PI. A randomized trial comparing intravenous immune globulin and plasma exchange in GuillainBarré syndrome. Dutch Guillain-Barré Study Group. N Engl J Med. 1992;326:1123-9.

12. Plasma Exchange/Sandoglobulin Guillain-Barré Syndrome Trial Group. Randomised trial of plasma exchange, intravenous immunoglobulin, and combined treatments in Guillain-Barré syndrome. Lancet. 1997;349:225-30.

13. Hughes RA, Swan AV, Raphael JC, Annane D, van Koningsveld $\mathrm{R}$, van Doorn PA. Immunotherapy for Guillain-Barré syndrome: a systematic review. Brain. 2007;130:2245-57.

14. van Koningsveld R, Schmitz PI, Meché FG, Visser LH, Meulstee J, van Doorn PA. Effect of methylprednisolone when added to standard treatment with intravenous immunoglobulin for Guillain-Barré syndrome: randomised trial. Lancet. 2004;363:192-6.

15. van Koningsveld R, Schmitz PI, Ang CW, Groen J, Osterhaus $\mathrm{AD}$, van der Meché FG, et al. Infections and course of disease in mild forms of Guillain-Barré syndrome. Neurology. 2002;58: $610-4$.

16. Durand MC, Porcher R, Orlikowski D, Aboab J, Devaux C, Clair $\mathrm{B}$, et al. Clinical and electrophysiological predictors of respiratory failure in Guillain-Barré syndrome: a prospective study. Lancet Neurol. 2006;5:1021-8.

17. van Koningsveld R, Steyerberg EW, Hughes RA, Swan AV, van Doorn PA, Jacobs BC. A clinical prognostic scoring system for Guillain-Barré syndrome. Lancet Neurol. 2007;6:589-94.

18. Farcas P, Avnun L, Frisher S, Herishanu YO, Wirguin I. Efficacy of repeated intravenous immunoglobulin in severe unresponsive Guillain-Barré syndrome. Lancet. 1997;350:1747.

19. Kuitwaard K, de Gelder J, Tio-Gillen AP, Hop WC, van Gelder T, van Toorenenbergen AW, et al. Pharmacokinetics of intravenous immunoglobulin and outcome in Guillain-Barré syndrome. Ann Neurol. 2009;66:597-603.

20. Cornblath DR, Hughes RA. Treatment for Guillain-Barré syndrome. Ann Neurol. 2009;66:569-70. 